\title{
Solitary Fibrous Tumor: An Uncommon Cause of Paraparesis and Sciatic Pain
}

\author{
Ana Vilaca ${ }^{\mathrm{a}, \mathrm{b}}$, Margarida Monteiro ${ }^{\mathrm{a}}$, Teresa Pimentel ${ }^{\mathrm{a}}$
}

\begin{abstract}
Solitary fibrous tumor (SFT) is a rare type of soft tissue neoplasia, usually seen in adults. They are mesenchymal neoplasias that appear to exhibit fibroblastic differentiation, classically described in the pleura, but can arise anywhere. They have a generally benign course; however, in rare cases, they may recur locally or at a distance. When surgery is not possible or in the presence of metastasis, chemotherapy or palliative radiotherapy may be therapeutic options. The patient was a 44-year-old man, a construction worker, healthy apart from a totally resected left pelvic SFT 3 years before. He was observed over the last several times in the emergency department (ED) by right sciatic pain and discharged. He had no history of trauma. A lumbar MRI was performed without alterations of relief. The worsening of the symptomatology, with commitment of the autonomous march, resorted him again to ED. Clinical features showed grade 2 strength with hypoesthesia and an aquiline reflex abolished in the right lower limb (MI) and strength grade $4+$ at the distal level of the left MI. There was a palpable rounded swelling on the posterior aspect of the right thigh $(5 \times 8 \mathrm{~cm})$, with an elastic and non-painful consistency at palpation. MRI of the neuro-axis showed no changes of relief. Pelvic CT showed swelling with soft tissue component, which invaded the iliac bilaterally and right sacroiliac joint, with lithic alteration. The histology of the pelvic lesion and thigh lesion revealed an SFT. Radiotherapy and then chemotherapy were performed. The patient died 1 year after the diagnosis. We report a fatal case of recurrence of SFT. The authors highlight the case because of its rarity and its "malignant" evolution. Long-term follow-up of these patients is required because of the risk of recurrence of these tumors and their uncertain biological behavior.
\end{abstract}

Keywords: Solitary fibrous tumor; Paraparesis; Sciatic pain

\section{Introduction}

Solitary fibrous tumor (SFT) is a rare type of soft tissue neo-

Manuscript accepted for publication May 17, 2017

anternal Medicine Department, Hospital Braga, Braga, Portugal ${ }^{b}$ Corresponding Author: Ana Vilaca, Travessa S. Pedro, no. 4, Este S. Pedro, Braga, Portugal. Email: ana.vilaca.rodrigues@gmail.com

doi: https://doi.org/10.14740/jmc2843w plasia, usually observed in adults. They are initially reported by Klemperer et al [1]. It is a mesenchymal tumor that appears to exhibit fibroblastic differentiation, classically described in the pleura, but extrathoracic SFT has also been reported [2]. Although sciatic pain is very common, extraspinal compression of the nerve is extremely rare. SFT can be diagnosed preoperatively only by needle biopsy [3] because it is a rare lesion and not easily recognizable. In addition, no specific tumor markers have been identified or images published for this tumor type. They have a generally benign course, being classified as having intermediate biological potential (rarely metastasizing) [4]. However, in rare cases, they may recur locally or at a distance. When surgery is not possible or in the presence of metastasis, chemotherapy or palliative radiotherapy may be therapeutic options.

\section{Case Report}

The patient was a 44-year-old man, a construction worker, healthy apart from a totally resected left pelvic SFT 3 years before. The patient presented at the emergency department with bilateral sciatic pain and gait disorder. He had progressive sciatic pain at the right for 11 months and on the left for 1 month that aggravated walking and sitting. He reported numbing and tingling of the right foot for 6 months. One month before, he developed constipation and effortful micturition, after a week, paresis of the right foot and leg, and later also his left foot. There is no history of trauma. Physical examination showed cafe-au-lait spots in the trunk (Fig. 1a), limbs (Fig. 1b) and axillary ephelides (Fig. 1c). There was also a palpable rounded swelling on the posterior aspect of the right thigh $(5 \times 8 \mathrm{~cm})$, with an elastic and non-painful consistency at palpation. Neurological examination disclosed an asymmetric paraparesis (right leg grade 2, left leg grade 4 MRC), normal deep tendon reflexes apart from abolished right aquilian reflex and indifferent right plantar response. He also showed reduced tactile and algic sensation distally in both legs. At this point, cauda equina syndrome due to compressive lesion was suspected, and spinal MRI was performed. With a normal MRI, an extraspinal cause was pursued. Pelvic CT scan was done that revealed a mass, which invaded the iliac bilaterally and right sacroiliac joint, with lithic alteration (Fig. 2a). The histology of the pelvic lesion and thigh lesion was compatible with bilateral SFT. The histological study revealed a cytological atypia and a high numerous of mitoses. Although the patient had no pulmonary 

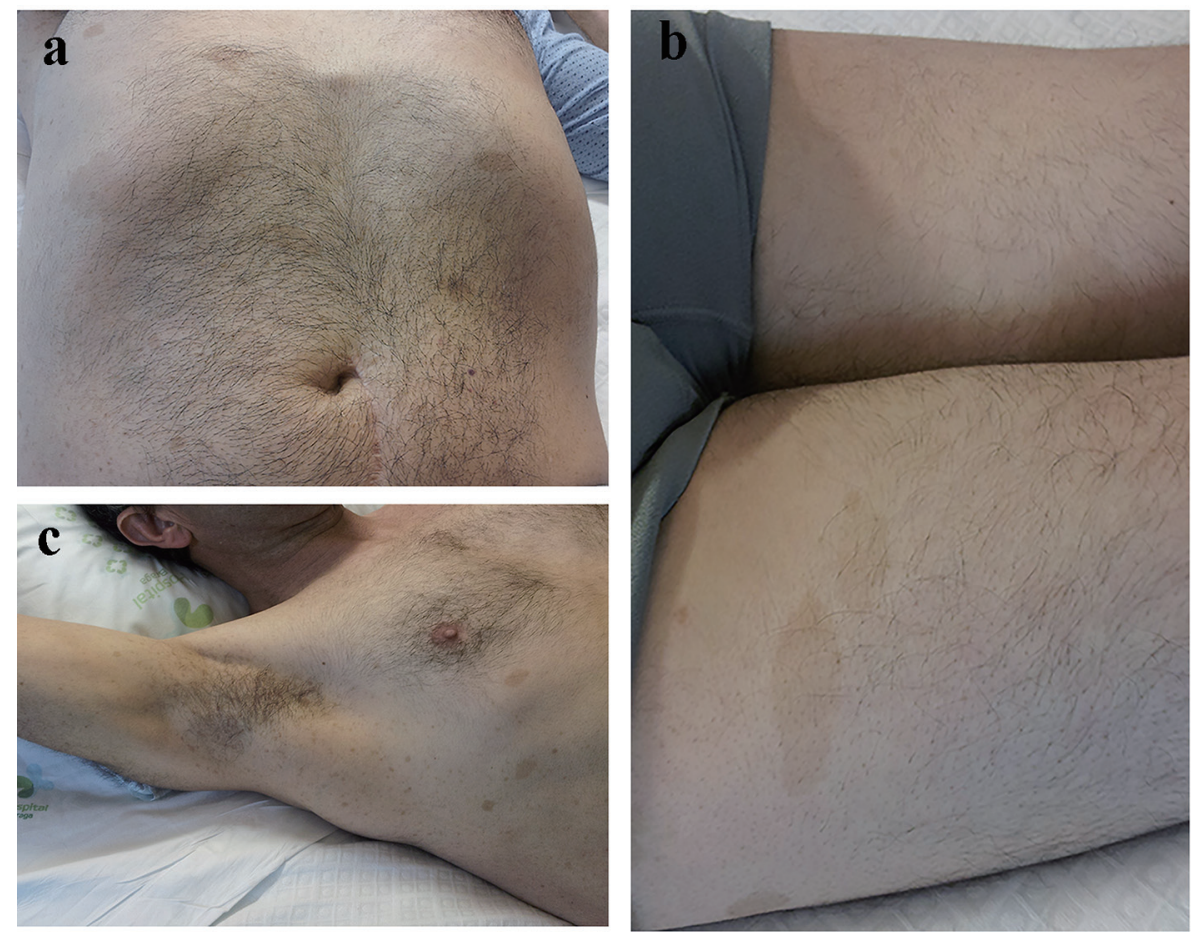

Figure 1. (a) Cafe-au-lait spots in the trunk. (b) Cafe-au-lait spots in the limbs. (c) Axillary ephelides.

symptoms, after the SFT diagnosis of the pelvic mass, a thoracic CT scan was also performed that showed a small peripheral nodule in the left upper lobe (Fig. 2b) and nodular densification area in the periphery posterior region of the apical region of the right lower lobe (Fig. 2c). After needle biopsy of the peripheral nodule in the left upper lobe histology, it was, indeed, compatible with SFT, with pleural involvement. As it was not possible to approach the pelvic mass surgically, due to vascular involvement, the patient initially received radiotherapy. Although the tumor did not respond, and after several sessions of radiotherapy, a therapeutic trial with chemotherapy was performed. No response had been seen and pelvic CTs and PET performed revealed growth of bilateral mass with invasion of adjacent organs: mesentery, sigmoid colon, intestinal adhesion and invasion of the bladder. The patient died 1 year after the diagnosis.

\section{Discussion}

The World Health Organization classification of soft tissue tumor in 2002 categorized extrapleural SFT as a fibroblastic/ myofibroblastic tumor of intermediate malignancy, which is defined as an ubiquitous mesenchymal tumor of possible fibroblastic type with a prominent hemangiopericytoma-like branching vascular pattern [5]. Although most cases are benign, the behavior of SFTs is unpredictable. Because of the overlapping of the histological diagnosis of SFT with other soft tissue tumors, its correct and precise pathological characterization necessitates experienced soft tissue pathologists to evaluate the specimen both for a proper diagnosis and for the detection of malignant features [6]. The malignant form causes an aggressive course of the disease, with local invasion, recurrence of disease and metastasis [7]. Pathological characteristics of malignant SFT include hypercellular lesions, cytological atypia, numerous mitoses (four or more mitoses per 10 high-power fields), tumor necrosis, and/or infiltrating margins [8]. Vallat-Decouvelaere et al reported malignancy in eight of 92 cases of extrathoracic SFT, including local recurrence or distal metastasis, in seven of which at least one of the typical pathological characteristics was observed in the primary tumor [9]. Various benign or malignant soft tissue tumors emerging from fat, muscle, and nerve may develop in the retroperitoneal region. Some retroperitoneal tumors are diagnosed because of clinical symptoms or image inspections. From the data collected we found no association between neurofibromatosis and SFT. In our case, progressive compression of the sacral plexus and sciatic nerve in the thigh was responsible for the symptoms and its appropriate investigation allowed the diagnosis. To our knowledge, this is the first report of sciatic pain as manifestation of SFT. However, little is still known about the genetic and molecular events responsible for the pathogenesis of SFT and how these may relate to clinically determined risk factors. A few immunohistochemically or molecular biomarkers, including p53, telomerase activity, cyclin expression, and $\mathrm{Ki} 67$, have been suggested to have prognostic significance [10]. Larger SFTs have been associated with more genomic copy number alterations than small tumors, suggesting a possible link to aggressive behavior. Future investigations to characterize potential biomarkers of SFT are needed [4]. As we said, SFT are rare tumors, especially in the advanced phase, as only a minority of patients will eventually recur after primary 

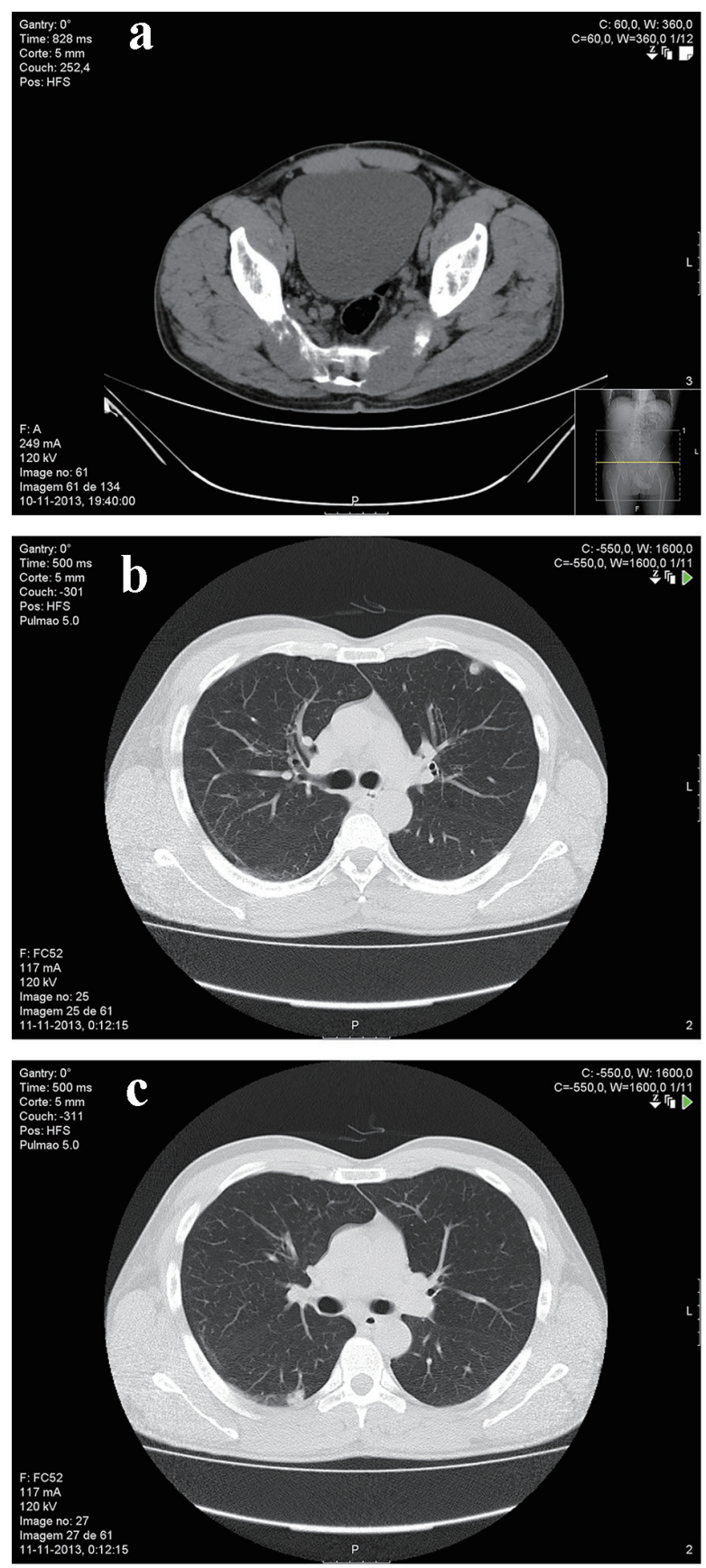

Figure 2. (A) Pelvic CT scan revealed a mass, which invaded the iliac bilaterally and right sacroiliac joint, with lithic alteration - histology of the pelvic lesion and thigh lesion was compatible with bilateral SFT. (b) Thoracic CT scan showed a small peripheral nodule in the left upper lobe. (c) Thoracic CT scan revealed nodular densification area in the periphery posterior region of the apical region of the right lower lobe.

surgical management [11]. As far as we know it is also the only report with such a malignant evolution, with disease progression, involving several organs, despite the treatments that were performed. Therefore, data regarding the actual effectiveness of systemic therapy in this setting are limited and stem from small retrospective studies. However, because the effi- cacy of standard chemotherapy is limited in STS, much hope has grown around the development of targeted therapies [11]. Furthermore, although anti-angiogenic agents have interesting activity in SFT, our data and those reported by others suggest that this subtype is only modestly more sensitive than other subtypes of sarcoma. This however would need to be assessed in a prospective trial which is greatly needed in these rare tumors. We underline the fact that the patient had no response either to radiotherapy or to chemotherapy.

\section{Author Contributions}

Ana Vilaca: substantial contributions to conception and design, acquisition of data, analysis and interpretation of data, drafting the article, critical revision of the article, and final approval of the version to be published. Margarida Monteiro: analysis and interpretation of data, critical revision of the article, and final approval of the version to be published. Teresa Pimentel: conception and design, analysis and interpretation of data, critical revision of the article, and final approval of the version to be published.

\section{Financial Support}

None.

\section{Conflicts of Interest}

Authors declare no conflicts of interest.

\section{Consent}

Patient informed consent was obtained.

\section{References}

1. Wang C, Manucha V, Faro S, Weaver M, Mukherjee AL. Fourth ventricular solitary fibrous tumor: a case report and review of the literature. J Med Case Rep. 2012;6:205.

2. Gomi K, Shimada K, Kajikawa S, Yazawa K, Shirota H, Nakamura T. A case of solitary fibrous tumor located in the retroperitoneum (in Japanese with English abstract). J Jpn Surg Assoc. 2009;70:2833-2838.

3. Schirosi L, Lantuejoul S, Cavazza A, Murer B, Yves Brichon P, Migaldi M, Sartori G, et al. Pleuro-pulmonary solitary fibrous tumors: a clinicopathologic, immunohistochemical, and molecular study of 88 cases confirming the prognostic value of de Perrot staging system and p53 expression, and evaluating the role of c-kit, BRAF, PDGFRs (alpha/beta), c-met, and EGFR. Am J Surg Pathol. 2008;32(11):1627-1642.

4. Demicco EG, Park MS, Araujo DM, Fox PS, Bassett RL, Pollock RE, Lazar AJ, et al. Solitary fibrous tumor: a clin- 
icopathological study of 110 cases and proposed risk assessment model. Mod Pathol. 2012;25(9):1298-1306.

5. Fletcher CDM, Unni K, Meryens F. World Health Organization classification of tumors, pathology and genetics, tumors of soft tissue and bone. Lyon: IARC Press; 2002:86-88.

6. Demirer Z, Zor M, Kurt B, Bozkurt Y, Yildirim I. Bilateral renal metastasis of an inguinal malignant solitary fibrous tumor, 9 years after primary surgical treatment. Med Princ Pract. 2012;21(6):585-587.

7. Teixeira, et al. Tumor Fibroso Solitario Retroperitoneal Volumoso. Revista da AMRIGS, Porto Alegre. 2014;58(3):237-239.

8. Tambo M, Fujimoto K, Miyake M, Hoshiyama F, Matsu- shita C, Hirao Y. Clinicopathological review of 46 primary retroperitoneal tumors. Int J Urol. 2007;14(9):785-788.

9. Tsushimi T, Yagi T, Tomozawa N, Ohnishi H. Retroperitoneal solitary fibrous tumor of the pelvis with pollakiuria: a case report. BMC Res Notes. 2012;5:593.

10. Miracco C, de Santi MM, Pacenti L, Schurfeld K, Laurini L, Pirtoli L, Luzi P, et al. Telomerase activity, Ki-67, cyclin D1 and A expression, and apoptosis in solitary fibrous tumors: additional features of a predictable course? Pathol Res Pract. 2001;197(7):475-481.

11. Levard A, Derbel O, Meeus P, Ranchere D, Ray-Coquard I, Blay JY, Cassier PA. Outcome of patients with advanced solitary fibrous tumors: the Centre Leon Berard experience. BMC Cancer. 2013;13:109. 